\title{
NUMERICAL ANALYSIS OF STOCHASTIC DIFFERENTIAL EQUATIONS WITH EXPLOSIONS
}

\author{
JUAN DÁVILA, JULIAN FERNÁNDEZ BONDER, PABLO GROISMAN, JULIO D. \\ ROSSI, AND MARIELA SUED
}

\begin{abstract}
Stochastic ordinary differential equations may have solutions that explode in finite or infinite time. In this article we design an adaptive numerical scheme that reproduces the explosive behavior. The time step is adapted according to the size of the computed solution in such a way that, under adequate hypotheses, the explosion of the solutions is reproduced.
\end{abstract}

\section{INTRODUCTION}

Consider the following stochastic differential equation (SDE):

$$
d x=b(x) d t+\sigma(x) d W,
$$

with $x(0)=z \in \mathbb{R}_{>0}$, where $b$ and $\sigma$ are smooth positive functions $\left(C^{1}\right.$ or even locally Lipschitz will be enough for our calculations) and $W$ is a (one dimensional) Wiener process defined on a given complete probability space $(\Omega, \mathcal{F}, \mathbb{P})$ with a filtration $\left\{\mathcal{F}_{t}\right\}_{t \geq 0}$ satisfying the usual conditions (i.e. it is right continuous and $\mathcal{F}_{0}$ contains all $\mathbb{P}$-null sets, [4]).

It is well known that stochastic differential equations like $(P)$ may explode in finite time. That is, trajectories may diverge to infinity as $t$ goes to some finite time $T$ that in general depends on the particular path.

The Feller Test for explosions (see $[4,6]$ ) gives a precise description in terms of $b, \sigma$ and $z$ of whether explosions in finite time occur with probability zero, positive or one. We review some well known facts about SDE with explosions in Section 2.

For example, if $b$ and $\sigma$ behave like powers at infinity, i.e.

$$
b(s) \sim s^{p}, \quad \sigma(s) \sim s^{q} \quad(s \rightarrow \infty),
$$

Key words and phrases. Explosion, stochastic differential equations, Numerical approximations.

Supported by Universidad de Buenos Aires under grant TX066, by ANPCyT PICT No. 03-05009, Fundación Antorchas Project 13900-5 and CONICET (Argentina). JFB is also supported by ANPCyT PICT No. 03-10608.

2000 Mathematics Subject Classification: 65C30, 65L20, 60H10. 
applying the Feller test one obtains that solutions to $(P)$ explode with probability one if $p>2 q \vee 1$. We use $f(s) \sim g(s)$ to mean that there exist constants $0<c<C$ such that $c g(s) \leq f(s) \leq C g(s)$ for large enough $s$. We also use $a \vee b=\max \{a, b\}, a \wedge b=\min \{a, b\}$.

The intuition behind this condition is that $p>2 q$ ensures that the asymptotic behavior of the solutions is governed by the drift term while $p>1$ impose the solution to grow up so fast that explodes in finite time, as in the deterministic case $(\sigma=0)$.

Stochastic differential equations like $(P)$ have been considered, for example, in fatigue cracking (fatigue failures in solid materials) with $b$ and $\sigma$ of power type, $[5,9]$ and so solutions may explode in finite time. This explosion time is generally random, depends on the particular sample path and corresponds to the time of ultimate damage or fatigue failure in the material.

Unfortunately explicit solvable SDEs are rare in practical applications, hence the importance of developing numerical methods to approximate them.

There are many numerical methods designed to deal with SDEs like $(P)$ when $b$ and $\sigma$ are assumed to be globally Lipschitz continuous. See for instance, the surveys $[2,7]$ and the book [5]. See also [3] where locally Lipschitz coefficients are considered. However, all of the cited work deal with globally defined solutions, and most of them with a constant time step. When dealing with explosive solutions these methods do not apply mainly because using a constant time step, produces approximations that are globally defined. Moreover, the convergence results are based on regularity assumptions of the solution in a fixed (deterministic) time interval $[0, \tau]$, these hypotheses are not available in our case.

The main purpose of this article is to develop an adaptive method that reproduces explosions of the solutions in case that it occurs, providing rigorous proofs of this fact.

We want to remark that even for deterministic problems the usual numerical methods are not well suited to reproduce explosions and therefore adaptive schemes have been developed, [1].

1.1. The numerical scheme. Let $h>0$ be the parameter of the method and let $\left\{X_{k}\right\}_{k \geq 1}=\left\{X_{k}^{h}\right\}_{k \geq 1}$ be the numerical approximation of $(P)$ given by the Euler-Maruyama method

$\left(P_{h}\right) \quad X_{k+1}=X_{k}+\tau_{k} b\left(X_{k}\right)+\sigma\left(X_{k}\right) \Delta W_{k}, \quad X_{0}=x(0)=z$,

where $\Delta W_{k}=W_{t_{k+1}}-W_{t_{k}}$ denotes the increment of the Wiener process in the interval $\left[t_{k}, t_{k+1}\right]$ and $\tau_{k}=t_{k+1}-t_{k}$. 
We define, for notational purposes, $X(t)$ as an interpolant of $X\left(t_{k}\right)=X_{k}$. For instance we can take $X(t) \equiv X_{k}$ for $t_{k} \leq t<t_{k+1}$ or $X(t)$ to be the linear interpolant of the values $X_{k}$.

Observe that the numerical approximation $X(t)$ is a well defined process up to time

$$
T_{h}:=\sum_{k=1}^{\infty} \tau_{k} .
$$

We say that a sample path $X(\cdot, \omega)$ of $\left(P_{h}\right)$ explodes in finite time if

$$
X(t, \omega) \rightarrow+\infty \quad\left(t \rightarrow T_{h}\right), \quad \text { and } \quad T_{h}(\omega)<\infty .
$$

If $b$ and $\sigma$ are globally Lipschitz continuous it is customary to take a constant time step $\tau_{k} \equiv h$ (see [5]). However when designing adaptive algorithms the time step $\tau_{k}$ has to be selected according to the computed solution $X_{k}$ and so it will be necessarily aleatory. Inspired by [1], we select the time steps $\tau_{k}$ according to the rule

$$
\tau_{k}=\frac{h}{b\left(X_{k}\right)} .
$$

Observe that by our selection of $\tau_{k}, T_{h}(\omega)<\infty$ implies $X(t) \nearrow+\infty$.

1.2. Main results. First, we prove convergence of the numerical approximations in compact (random) intervals where the solution and the numerical approximation are bounded. For this theorem the time steps $\tau_{k}$ only need to be $\mathcal{F}_{t_{k}}-$ measurable and verify $\tau_{k} \leq C h$ but are otherwise arbitrary.

Theorem 1.1 (Convergence of the numerical scheme). Let $x(\cdot)$ be the solution of $(P)$ and $X(\cdot)$ its EM approximation given by $\left(P_{h}\right)$. Fix a time $\bar{\tau}>0$ and a constant $M>0$. Consider the stopping times given by $\tau:=\bar{\tau} \wedge R^{M}$ and $\tau_{h}:=\bar{\tau} \wedge R_{h}^{2 M}$, where $R^{M}:=\inf \{t:|x(t)| \geq M\}$ and $R_{h}^{M}:=\inf \{t:|X(t)| \geq M\}$. Then

$$
\lim _{h \rightarrow 0} \mathbb{E}\left[\sup _{0 \leq t \leq \tau \wedge \tau_{h}}|x(t)-X(t)|^{2}\right]=0 .
$$

Observe that if the sample paths are uniformly bounded this is a standard convergence theorem (see for example $[3,5]$ ). However, in case that there exists solutions that explode in finite time we prove convergence of the numerical scheme in regions where they are bounded. We do not expect convergence in bigger regions.

However, if we weaken the notion of convergence, we can prove that the computed solution converges to the continuous one in any interval where the continuous solution remains bounded. More precisely, we have 
Corollary 1.2. With the same assumptions and notation as in Theorem 1.1 , for every $\varepsilon>0$ and every $0 \leq \alpha<\frac{1}{2}$,

$$
\mathbb{P}\left(\sup _{0<t<R^{M}}|x(t)-X(t)|>\varepsilon h^{\alpha}\right) \rightarrow 0 \quad \text { as } h \rightarrow 0 .
$$

Next we assume a specific behavior on the coefficients in $(P)$ to have explosions with probability one. The precise assumptions on $b, \sigma$ are: there exist positive constants $\kappa_{1}, \kappa_{2}$ such that

(A) $\quad \kappa_{1} \leq \sigma^{2}(s) \leq \kappa_{2} b(s), \quad b$ is nondecreasing and $\quad \int_{0}^{\infty} \frac{1}{b(s)} d s<\infty$.

Remark 1.1. By means of the Feller Test, one can check that under assumption (A) solutions to $(P)$ explode with probability one. These assumptions are actually stronger than the ones required by the Feller Test. Recall that the solution of the (deterministic) differential equation $\bar{x}^{\prime}(t)=b(\bar{x}(t))$ with $\bar{x}(0)=z \in \mathbb{R}_{>0}$ explodes in finite time if and only if $\int_{z}^{+\infty} \frac{1}{b(s)} d s<\infty$.

Next, we analyze the asymptotic behavior of the solutions to $\left(P_{h}\right)$ and show that it agrees with the behavior of the solutions to $(P)$. This is our main result.

Theorem 1.3. Assume (A). Then

(1) For every initial datum $z>0, X(\cdot)$ explodes in finite time with probability one.

(2) We have,

$$
\lim _{k \rightarrow \infty} \frac{X\left(t_{k}\right)}{h k}=1 \quad \text { a.s. }
$$

Moreover, for any $\alpha>1$ there exits $k_{0}=k_{0}(\omega)$ such that, for every $k \geq k_{0}$ there holds

$$
\sum_{j=k}^{\infty} \frac{h}{b(\alpha h j)} \leq T_{h}-t_{k} \leq \sum_{j=k}^{\infty} \frac{h}{b\left(\alpha^{-1} h j\right)} .
$$

(3) If b has regular variation at infinity (see Definition 4.3),

$$
\lim _{k \rightarrow \infty} \frac{T_{h}-t_{k}}{\int_{X_{k}}^{\infty} \frac{1}{b(s)} d s}=1 \quad \text { a.s. }
$$

(4) In addition, for every $h>0$, there holds $h / b(z) \leq T_{h}<+\infty$ a.s and for every $L>0, \mathbb{P}\left(T_{h}>L\right)>0$.

Remark 1.2. Observe that (3) gives the precise asymptotic behavior of the numerical solution near the explosion time. For example, if $b(s) \sim s^{p}$, the explosion rate given by $(3)$ is

$$
X\left(t_{k}\right)\left(T_{h}-t_{k}\right)^{1 /(p-1)} \rightarrow\left(\frac{1}{p-1}\right)^{\frac{1}{p-1}}, \quad\left(t_{k} \rightarrow T_{h}\right) .
$$


This is the behavior of solutions to the deterministic ODE $d \bar{x}(t)=\bar{x}^{p}(t) d t$.

Finally, we analyze the convergence of the stopping times considered in Theorem 1.1 to the explosion time of the continuous problem.

Theorem 1.4. Assume (A). Then, for any $\varepsilon>0$,

$$
\lim _{M \rightarrow+\infty} \lim _{h \rightarrow 0} \mathbb{P}\left(\left|R_{h}^{M}-T\right|>\varepsilon\right)=0 .
$$

This last Theorem is useful in actual computations of the explosion time for $(P)$. See Section 5 .

The rest of the paper is organized as follows: in Section 2 we review some well known facts about the continuous problem $(P)$. In section 3 we deal with some measurability properties of the numerical scheme and prove the convergence results, Theorem 1.1 and Corollary 1.2. In Section 4 we analyze the numerical scheme $\left(P_{h}\right)$ and prove Theorem 1.3. In Section 5 we prove Theorem 1.4 that is the key point for approximate the (continuous) explosion time $T$. Finally, in section 6 , we show some numerical experiments.

\section{The COntinuous EQuation}

In this section we review some results concerning the behavior of solutions to $(P)$ as $t \nearrow T$, the explosion time. These results can be found, for instance, in $[4,8]$.

Let $s: \mathbb{R} \rightarrow \mathbb{R}$ be the scale function for $(P)$ given by

$$
s(z)=0, \quad s^{\prime}(\xi)=\exp \left[-\int_{0}^{\xi} 2 b(t) \sigma(t)^{-2} d t\right] .
$$

Then, if $y(t)=s(x(t))$, we have

$$
d y=\bar{\sigma}(y) d W
$$

where $\bar{\sigma}=\left(s^{\prime} \sigma\right) \circ s^{-1}$. Solutions to $(2.1)$ are globally defined. Observe that $x$ explodes in finite time if and only if

$$
\ell:=s(+\infty)<+\infty \text {. }
$$

We can obtain a weak solution to $(2.1)$ by time change. In fact, let $B(t)$ be a standard Brownian motion and define

$$
A(t)=\int_{0}^{t} \bar{\sigma}(B(u))^{-2} d u,
$$

and let $\gamma$ be the inverse of $A$, then

$$
y(t)=B(\gamma(t))
$$

is a weak solution of $(2.1)$. 
Let

$$
T_{\ell}:=\inf \{t>0: B(t)=\ell\}
$$

Therefore

$$
T=A\left(T_{\ell}\right)=\int_{0}^{T_{\ell}} \bar{\sigma}^{-2}(B(u)) d u,
$$

is the explosion time.

To describe the behavior of $x(t)$ near the explosion time $T$, we have to study the behavior of $B(t)$ when $t$ is close to $T_{\ell}$. To this end we define

$$
R(t):=\ell-B\left(T_{\ell}-t\right), \quad 0 \leq t \leq T_{\ell}
$$

Then $R(t)$ is a Bessel process, i.e. $R(t) \stackrel{\mathcal{L}}{=} \operatorname{BES}(3)$.

Combining these assertions, we get

$y(T-\varepsilon)=B(\gamma(T-\varepsilon))=B\left(T_{\ell}-\left(T_{\ell}-\gamma(T-\varepsilon)\right)\right)=\ell-R\left(T_{\ell}-\gamma(T-\varepsilon)\right)$.

So we arrive at

$$
x(T-\varepsilon)=s^{-1}(y(T-\varepsilon))=s^{-1}\left(\ell-R\left(T_{\ell}-\gamma(T-\varepsilon)\right)\right) .
$$

Therefore, we have found the asymptotic behavior for the solution $x$ to $(P)$ near the explosion time $T$. Moreover, $(2.2)$ gives an "explicit" formula for the explosion time $T$ of weak solutions to $(P)$.

\section{Convergence of the numerical scheme}

We begin this section by showing some measurability properties of the numerical scheme.

Lemma 3.1. With the notation of Section 1.2, $\left\{t_{k}\right\}_{k \geq 1}$ are stopping times and each $\tau_{k}$ is $\mathcal{F}_{t_{k}}$-measurable.

Proof. We just observe that

$$
t_{k+1}=t_{k}+\tau_{k}=t_{k}+\frac{h}{b\left(X_{k}\right)} .
$$

Assume $t_{k}$ is a stopping time. Then $X_{k}$ (and hence $\tau_{k}$ ) is $\mathcal{F}_{t_{k}}$-measurable. Since $\tau_{k}$ is positive, $t_{k+1}$ is also a stopping time. The case $k=0$ holds since $t_{0}$ is the constant $h / b(z)$.

Now we prove the main result of the section. Recall that this result and the subsequent proposition and corollary hold true for any choice of time steps $\tau_{k}$ if they are $\mathcal{F}_{t_{k}}$-measurables and $\tau_{k} \leq C h$. 
Proof of Theorem 1.1. First, we truncate the functions $b(x)$ and $\sigma(x)$ in such a way that they are globally Lipschitz, bounded and coincide with the original $b(x)$ and $\sigma(x)$ for values of $x$ with $|x| \leq 2 M$. i.e. we consider

$$
\bar{b}(x)= \begin{cases}b(x) & \text { if }|x| \leq 2 M \\ b(2 M) & \text { if } x \geq 2 M \\ b(-2 M) & \text { if } x \leq-2 M\end{cases}
$$

and

$$
\bar{\sigma}(x)= \begin{cases}\sigma(x) & \text { if }|x| \leq 2 M \\ \sigma(2 M) & \text { if } x \geq 2 M \\ \sigma(-2 M) & \text { if } x \leq-2 M .\end{cases}
$$

Let $y$ and $Y$ be the solutions of

$$
\begin{aligned}
& d y=\bar{b}(y) d t+\bar{\sigma}(y) d W, \quad y(0)=z, \\
& Y_{k+1}=Y_{k}+\tau_{k} \bar{b}\left(Y_{k}\right)+\bar{\sigma}\left(Y_{k}\right) \Delta W_{k}, \quad Y(0)=z,
\end{aligned}
$$

respectively.

From [5] we have

$$
\mathbb{E}\left[\sup _{0 \leq t \leq \bar{\tau}}|y-Y|^{2}\right] \rightarrow 0, \quad \text { as } h \rightarrow 0
$$

Recalling that $\tau:=\bar{\tau} \wedge R^{M}$ and $\tau_{h}:=\bar{\tau} \wedge R_{h}^{2 M}$ we have that $x(t)=y(t)$ and $X(t)=Y(t)$ if $0 \leq t \leq \tau \wedge \tau_{h}$. Hence

$$
\mathbb{E}\left[\sup _{0 \leq t \leq \tau \wedge \tau_{h}}|x-X|^{2}\right]=\mathbb{E}\left[\sup _{0 \leq t \leq \tau \wedge \tau_{h}}|y-Y|^{2}\right] \leq \mathbb{E}\left[\sup _{0 \leq t \leq \bar{\tau}}|y-Y|^{2}\right] .
$$

This implies the result.

Remark 3.1. Observe that, in fact, the results in [5] gives

$$
\mathbb{E}\left[\sup _{0 \leq t \leq \bar{\tau}}|y-Y|^{2}\right] \leq C h
$$

so in our case, we also obtain

$$
\mathbb{E}\left[\sup _{0 \leq t \leq \tau \wedge \tau_{h}}|x-X|^{2}\right] \leq C h .
$$

What one really wants in Theorem 1.1 is convergence of the numerical scheme without any assumptions on the computed solution $X$. Unfortunately, we are not able to prove convergence in square mean without this hypothesis. However, we are able to prove convergence in probability without any further assumption on $X$. To this end we need the following Proposition.

Proposition 3.2. Let $R^{M}$ and $R_{h}^{M}$ be as in Theorem 1.1 and $M>0$, then we have 
(1) $\mathbb{P}\left(R^{M} \geq R_{h}^{2 M}\right) \rightarrow 0 \quad$ as $h \rightarrow 0$.

(2) $\mathbb{P}\left(R_{h}^{M} \geq R^{2 M}\right) \rightarrow 0$ as $h \rightarrow 0$.

Proof. First we prove (1). Let $\varepsilon>0$. As $T<\infty$ a.s., we can take $\bar{\tau}$ such that

$$
\mathbb{P}(T>\bar{\tau})<\frac{\varepsilon}{2} .
$$

Now, with the notation of the Theorem 1.1 we have that

$$
\begin{aligned}
\mathbb{P}\left(R^{M} \geq R_{h}^{2 M}\right) & \leq \mathbb{P}(T>\bar{\tau})+\mathbb{P}\left(\sup _{0<t<\tau} Y \geq 2 M\right) \\
& <\frac{\varepsilon}{2}+\mathbb{P}\left(\sup _{0<t<\tau} Y \geq 2 M\right) \\
& <\varepsilon
\end{aligned}
$$

if $h$ is small enough. In fact, by Tchebychev inequality,

$\mathbb{P}\left(\sup _{0<t<\tau} Y \geq 2 M\right) \leq \mathbb{P}\left(\sup _{0 \leq t \leq \tau}|y-Y|>M\right) \leq \frac{1}{M^{2}} \mathbb{E}\left[\sup _{0 \leq t \leq \bar{\tau}}|y-Y|^{2}\right]<\frac{\varepsilon}{2}$,

from where (1) follows.

To prove (2), taking $\bar{\tau}$ as before, we have

$$
\begin{aligned}
\mathbb{P}\left(R_{h}^{M} \geq R^{2 M}\right) & \leq \mathbb{P}(T \geq \bar{\tau})+\mathbb{P}\left(R_{h}^{M} \geq R^{2 M}, T<\bar{\tau}\right) \\
& \leq \frac{\varepsilon}{2}+\mathbb{P}\left(\sup _{0<t<\bar{\tau} \wedge R_{h}^{M}} y \geq 2 M\right) .
\end{aligned}
$$

The proof follows as in (1).

Now, combining Theorem 1.1 and Proposition 3.2, We can get rid of the boundedness assumption on $X$ by weakening the notion of convergence.

Proof of Corollary 1.2. First, take $\bar{\tau}>0$ such that

$$
\mathbb{P}(T>\bar{\tau})<\frac{\delta}{2}
$$

Then, if $\tau=\bar{\tau} \wedge R^{M}$, by Tchebychev's inequality,

$$
\begin{aligned}
\mathbb{P}\left(\sup _{0<t<\tau}|x(t)-X(t)|>\varepsilon h^{\alpha}\right) \leq & \mathbb{P}\left(\sup _{0<t<\tau}|x(t)-X(t)|>\varepsilon h^{\alpha}, R^{M}<R_{h}^{2 M}\right) \\
& +\mathbb{P}\left(R^{M} \geq R_{h}^{2 M}\right) \\
\leq & \frac{1}{h^{2 \alpha} \varepsilon^{2}} \mathbb{E}\left[\sup _{0<t<\tau \wedge \tau_{h}}|x(t)-X(t)|^{2}\right] \\
& \quad+\mathbb{P}\left(R^{M} \geq R_{h}^{2 M}\right) \\
\leq & \frac{h^{1-2 \alpha}}{\varepsilon}+\mathbb{P}\left(R^{M} \geq R_{h}^{2 M}\right) \rightarrow 0
\end{aligned}
$$


as $h \rightarrow 0$. Therefore, for $h$ small (depending on $\delta$ )

$$
\mathbb{P}\left(\sup _{0<t<R^{M}}|x(t)-X(t)|>\varepsilon h^{\alpha}\right)<\delta .
$$

This finishes the proof.

\section{Explosions IN THE NUMERICAL SCHEME}

In this section we prove that for almost every $\omega \in \Omega, X(t)$ explodes in finite time $T_{h}(\omega)$.

To begin with, let us recall an auxiliary lemma.

Lemma 4.1 ([4], Chapter 2). Let $\left\{\mathcal{F}_{t}\right\}$ be the filtration generated by the Wiener process $W(\cdot)$ and $S$ a stopping time of $\left\{\mathcal{F}_{t}\right\}$. Assume $\tau$ is a random variable $\mathcal{F}_{S}$-measurable. Then for every Borel set $A$ it holds

$$
\mathbb{P}\left(W(S+\tau)-W(S) \in A \mid \mathcal{F}_{S}\right)=\int_{A} \frac{1}{\sqrt{2 \pi \tau}} e^{-\frac{x^{2}}{2 \tau}} d x
$$

Furthermore,

$$
\left.\frac{W(S+\tau)-W(S)}{\sqrt{\tau}}\right|_{\mathcal{F}_{S}}
$$

is a standard normal random variable and hence (4.1) holds without conditioning.

Next, we prove a technical lemma that is the key point in the proof of Theorem 1.3. This lemma allows us to control the effect of the diffusion in the numerical approximations of $(P)$.

Lemma 4.2. Let $Y_{k}=\sum_{j=1}^{k} \sigma\left(X_{j}\right) \Delta W_{j}$. Then

$$
\lim _{k \rightarrow \infty} \frac{Y_{k}}{k}=0 \quad \text { a.s. }
$$

Proof. Let

$$
Z_{j}:=\frac{\Delta W_{j}}{\sqrt{\tau_{j}}}=\frac{W_{t_{j}+\tau_{j}}-W_{t_{j}}}{\sqrt{\tau_{j}}} \quad \text { and } \quad a_{j}:=\sigma\left(X_{j}\right) \sqrt{\tau_{j}}=\sqrt{h} \frac{\sigma\left(X_{j}\right)}{\sqrt{b\left(X_{j}\right)}} .
$$

Then $Y_{k}=\sum_{j=1}^{k} a_{j} Z_{j}$. Observe that $a_{j}$ are uniformly bounded by assumption (A). In order to prove that $Y_{k} / k$ goes to zero, we use Tchebychev's inequality combined with Borel-Cantelli's Lemma. So we need to show that

$$
\sum_{k=1}^{\infty} \frac{\mathbb{E}\left[Y_{k}^{4}\right]}{k^{4}}<\infty
$$

Observe that $Z_{j}$ is independent of $\mathcal{F}_{t_{j}}$ and is normally distributed, according Lemma 4.1. Then, if $i \neq j, r$ or $l$, conditioning we obtain

$$
\mathbb{E}\left[Z_{i} Z_{j} Z_{r} Z_{l}\right]=0
$$


Moreover

$$
\mathbb{E}\left[Z_{i}^{2} Z_{j}^{2}\right]=1 \quad(i \neq j) \quad \text { and } \quad \mathbb{E}\left[Z_{i}^{4}\right]=3 .
$$

Hence

$$
\begin{aligned}
\mathbb{E}\left[\left(\sum_{j=1}^{k} Z_{j}\right)^{4}\right] & =\sum_{j=1}^{k} \mathbb{E}\left[Z_{j}^{4}\right]+3 \sum_{\substack{i, j=1 \\
i \neq j}}^{k} \mathbb{E}\left[Z_{i}^{2} Z_{j}^{2}\right] \\
& =3 k+3\left(k^{2}-k\right) \\
& =3 k^{2}
\end{aligned}
$$

Taking into account that $a_{j}$ is $\mathcal{F}_{t_{j}}$-measurable, proceeding in the same way with $a_{j} Z_{j}$ we obtain $\mathbb{E}\left[Y_{k}^{4}\right] \leq 3\left(\kappa_{2} h k\right)^{2}$ to get the desired result.

Now we use this lemma to prove that solutions to the numerical scheme explode with probability one and to find the rate of explosion. We are going to use the following

Definition 4.3. We say that a function $f: \mathbb{R} \rightarrow \mathbb{R}$ has regular variation at infinity if there exist $p>0$ such that for every positive $\alpha$,

$$
\lim _{s \rightarrow+\infty} \frac{f(\alpha s)}{f(s)}=\alpha^{p}
$$

Proof of Theorem 1.3. Since $X_{k}=z+h k+Y_{k-1}$, by Lemma 4.2,

$$
\frac{X_{k}}{h k}=\frac{z}{h k}+1+\frac{Y_{k-1}}{h k} \rightarrow 1, \quad \text { a.s. } \quad \text { as } k \rightarrow \infty .
$$

To prove that explosion occurs, it rest to show that with probability one, $T_{h}=\sum \tau_{j}<\infty$. To this end observe that, by (4.2) for every $\alpha>1$, there exist $k_{0}=k_{0}(\omega)$ such that,

$$
\begin{aligned}
\sum_{k=k_{0}}^{\infty} \tau_{k} & =\sum_{k=k_{0}}^{\infty} \frac{h}{b\left(X_{k}\right)} \leq \sum_{k=k_{0}}^{\infty} \frac{h}{b\left(\alpha^{-1} h k\right)} \leq \int_{k_{0}-1}^{\infty} \frac{h}{b\left(\alpha^{-1} h s\right)} d s \\
& =\alpha \int_{\alpha^{-1} h\left(k_{0}-1\right)}^{\infty} \frac{1}{b(u)} d u \leq \alpha \int_{X_{k_{0}-1}}^{\infty} \frac{1}{b(u)} d u<+\infty, \quad \text { a.s. }
\end{aligned}
$$

This proves (1).

In order to proof of (2) we observe that the computations performed above gives

$$
T_{h}-t_{k} \leq \sum_{j=k}^{\infty} \frac{h}{b\left(\alpha^{-1} h j\right)} \leq \alpha \int_{X_{k}}^{\infty} \frac{1}{b(u)} d u .
$$

In the same way, we can obtain the reverse inequality,

$$
T_{h}-t_{k} \geq \sum_{j=k}^{\infty} \frac{h}{b(\alpha h j)} \geq \frac{1}{\alpha} \int_{X_{k+1}}^{\infty} \frac{1}{b(u)} d u,
$$


for $k=k(\omega)$ large enough.

To prove (3), just observe that regular variation at infinity of $b$ implies that

$$
B(t):=\int_{t}^{\infty} \frac{1}{b(u)}, d u
$$

has regular variation at infinity with the same exponent. Therefore, as $B$ is increasing,

$$
\frac{B\left(X_{k}\right)}{B\left(X_{k+1}\right)}=\frac{B\left(\frac{X_{k}}{X_{k+1}} X_{k+1}\right)}{B\left(X_{k+1}\right)} \leq \frac{B\left(\alpha X_{k+1}\right)}{B\left(X_{k+1}\right)} \rightarrow \alpha^{p} \quad \text { as } k \rightarrow \infty
$$

for any $\alpha>1$. Therefore

$$
\limsup _{k \rightarrow \infty} \frac{B\left(X_{k}\right)}{B\left(X_{k+1}\right)} \leq 1
$$

Analogously,

$$
\liminf _{k \rightarrow \infty} \frac{B\left(X_{k}\right)}{B\left(X_{k+1}\right)} \geq 1
$$

It remains to show (4), but this follows from the fact that for any $K>0$,

$$
\mathbb{P}\left(\max _{1 \leq j \leq K} X_{j}<2 z\right)>0 .
$$

Hence, if $K$ is such that $K h / b(2 z)>L$, we obtain

$$
\mathbb{P}\left(T_{h}>L\right) \geq \mathbb{P}\left(\max _{1 \leq j \leq K} X_{j}<2 z\right)>0
$$

Moreover $T_{h} \geq \tau_{1}=h / b(z)$. The proof is now complete.

\section{Approximation of the explosion time}

In this section we prove Theorem 1.4. Observe that in numerical simulations $R_{h}^{M}$ can be easily computed. This fact together with Theorem 1.4 allows us to construct the numerical approximation of $T$ given in the next section.

Proof of Theorem 1.4. We proceed as follows, for $\varepsilon>0$ we have

$$
\mathbb{P}\left(\left|R_{h}^{M}-T\right|>\varepsilon\right)=\mathbb{P}\left(R_{h}^{M}-T>\varepsilon\right)+\mathbb{P}\left(R_{h}^{M}-T<-\varepsilon\right)=I+I I .
$$

We first show that $I$ goes to zero as $h \rightarrow 0$ for any fixed $M$. In fact, as $R^{2 M}<T$

$$
I \leq \mathbb{P}\left(R_{h}^{M}-R^{2 M}>\varepsilon\right) \leq \mathbb{P}\left(R_{h}^{M}>R^{2 M}\right) \rightarrow 0,
$$

as $h \rightarrow 0$, by Proposition 3.2. 
For the second term, we have

$$
\begin{aligned}
I I & \leq \mathbb{P}\left(R^{M / 2}-R_{h}^{M}>\varepsilon / 2\right)+\mathbb{P}\left(T-R^{M / 2}>\varepsilon / 2\right) \\
& \leq \mathbb{P}\left(R^{M / 2}>R_{h}^{M}\right)+\mathbb{P}\left(\left|T-R^{M / 2}\right|>\varepsilon / 2\right) \rightarrow 0
\end{aligned}
$$

by Proposition 3.2 and since $R^{M / 2} \rightarrow T$ a.s. $(M \rightarrow+\infty)$.

This completes the proof.

\section{Numerical EXPERIMENTS}

In this section we present some numerical examples to illustrate the theory set forth in the previous sections. All the experiments are computed with

$$
b(\xi)=|\xi|^{1.1}+0.1, \quad \sigma(\xi)=\sqrt{|\xi|^{1.1}+0.1}, \quad z=10 .
$$

The increments of the Wiener process have been generated with the randn routine of MATLAB.

In Figure 1 we show some sample paths of the solution. We stop the algorithm when the computed solution reaches $M=10^{5}$.

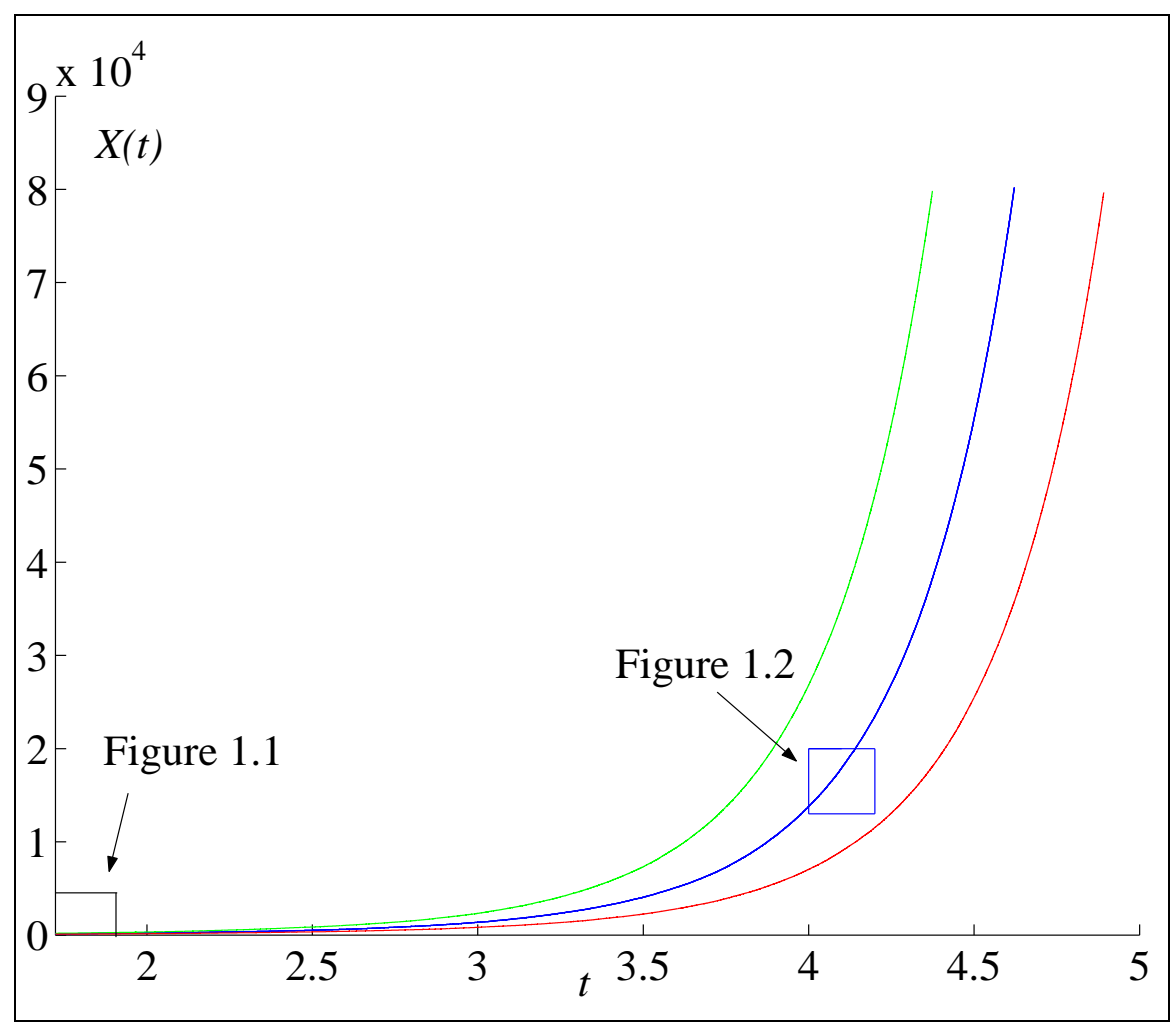

Figure 1: Three sample paths with explosions 


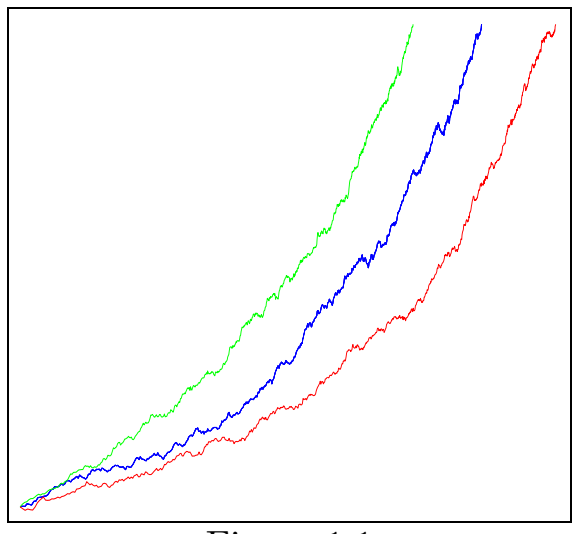

Figure 1.1

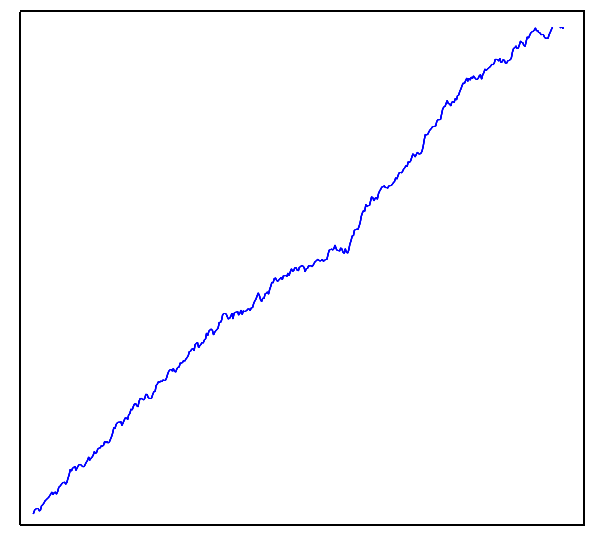

Figure 1.2

In Figure 2 we show the ratio $X_{k} / h k$, and observe that it converges to 1 as predicted by our result.

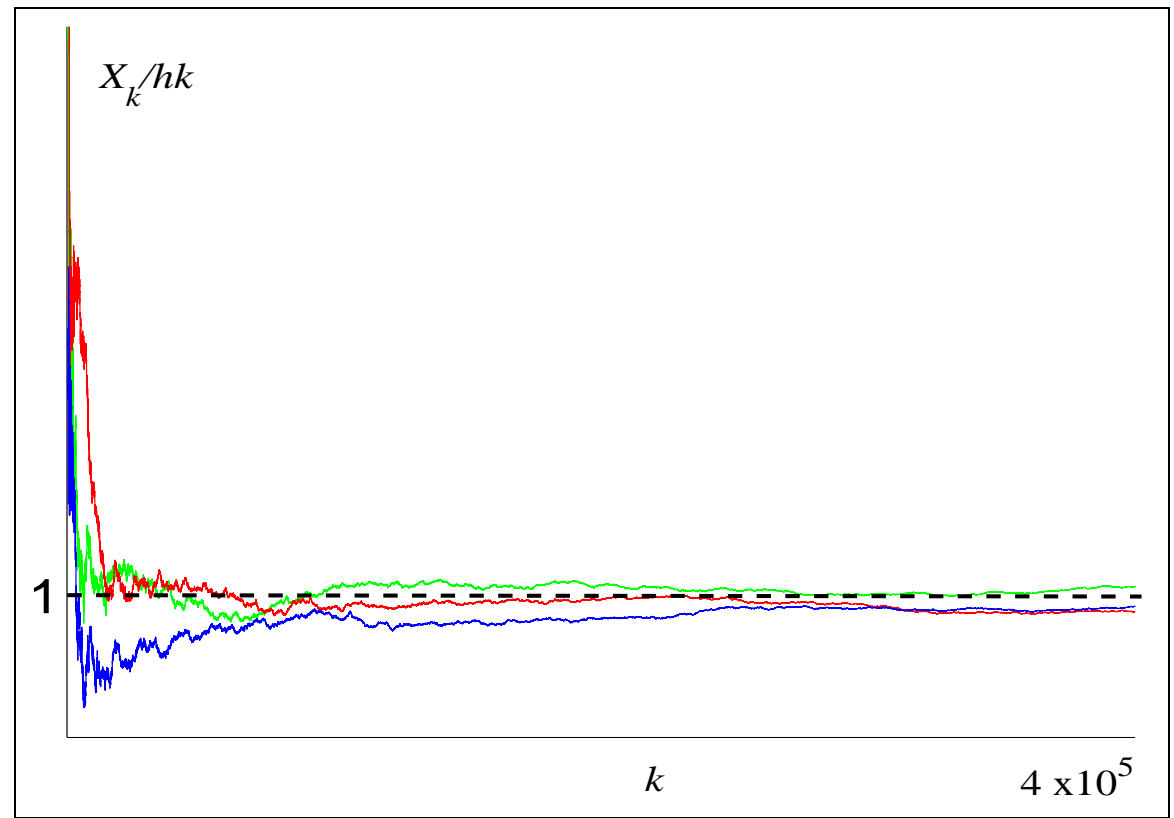

Figure 2: $X_{k} / h k \rightarrow 1$ a.s.

Finally, Figure 3 shows the kernel density estimation of $R_{h}^{M}$ for different choices of $h$. As proved in Theorem $1.4 R_{h}^{M}$ converges in probability to $T$.

We have used 1000 sample paths for each estimator. The values of $h$ taken in each estimator were $h=1, h=0.5$ and $h=0.1$. Observe that in each case, the largest time step taken were $\tau_{1} \simeq 0.08, \tau_{1} \simeq 0.04$ and $\tau_{1} \simeq 0.008$ respectively. 


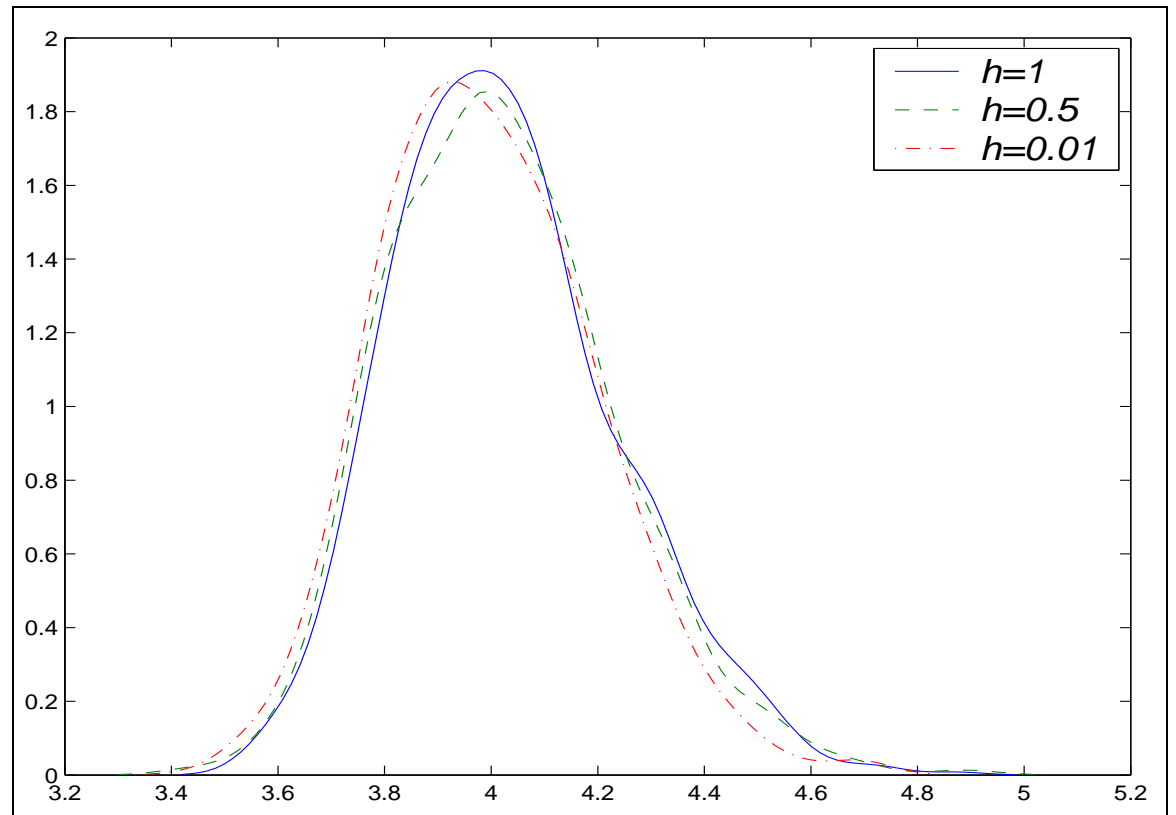

Figure 3: The kernel density estimator of $R_{h}^{M}$ for different values of $h$.

Acknowledgements. We like to thank Jean Bertoin and Pablo Ferrari for several interesting discussions that help us to improve this article. JFB and PG like to thank the hospitality received at the University of Sao Paulo, where part of the work has been done. JD would like to thank the invitation and hospitality at the Department of Mathematics at the University of Buenos Aires

\section{REFERENCES}

[1] Gabriel Acosta, Ricardo Durán, and Julio D. Rossi. An adaptive time step procedure for a parabolic problem with blow-up. Computing, 68(4):343-373, 2002.

[2] Desmond J. Higham. An algorithmic introduction to numerical simulation of stochastic differential equations. SIAM Rev., 43(3):525-546 (electronic), 2001.

[3] Desmond J. Higham, Xuerong Mao, and Andrew M. Stuart. Strong convergence of Euler-type methods for nonlinear stochastic differential equations. SIAM J. Numer. Anal., 40(3):1041-1063 (electronic), 2002.

[4] Ioannis Karatzas and Steven E. Shreve. Brownian motion and stochastic calculus, volume 113 of Graduate Texts in Mathematics. Springer-Verlag, New York, second edition, 1991.

[5] Peter E. Kloeden and Eckhard Platen. Numerical solution of stochastic differential equations, volume 23 of Applications of Mathematics (New York). Springer-Verlag, Berlin, 1992.

[6] H. P. McKean, Jr. Stochastic integrals. Probability and Mathematical Statistics, No. 5. Academic Press, New York, 1969.

[7] Eckhard Platen. An introduction to numerical methods for stochastic differential equations. In Acta numerica, 1999, volume 8 of Acta Numer., pages 197-246. Cambridge Univ. Press, Cambridge, 1999. 
[8] L. C. G. Rogers and David Williams. Diffusions, Markov processes, and martingales. Vol. 2. Cambridge Mathematical Library. Cambridge University Press, Cambridge, 2000. Itô calculus, Reprint of the second (1994) edition.

[9] K. Sobczyk and B. F. Spencer, Jr. Random fatigue. Academic Press Inc., Boston, MA, 1992. From data to theory.

J. DÁVILA

Departamento de Ingeniería Matemática, Universidad de Chile, CMM

Blanco Encalada 2120, 5to piso, Santiago, Chile.

E-mail address: jdavila@dim.uchile.cl

J. Fernández Bonder and J.D. Rossi

Departamento de Matemática, FCEyn, Universidad de Buenos Aires, Pabellón I, Ciudad Universitaria (1428), Buenos Aires, Argentina.

E-mail address: jfbonder@dm.uba.ar, jrossi@dm.uba.ar

P. Groisman and M. Sued

Instituto de CÁlculo, FCEyN, Universidad de Buenos Aires,

Pabellón II, Ciudad Universitaria (1428), Buenos Aires, Argentina.

E-mail address: pgroisma@dm.uba.ar, msued@dm.uba.ar 